\title{
Coulomb Drag between Quantum Wires
}

\author{
Rochus Klesse* and Ady Stern \\ Department of Condensed Matter Physics, The Weizmann Institute of Science, Rehovot 76100, Israel
}

(July 5, 2021)

\begin{abstract}
We study Coulomb drag in a pair of parallel one-dimensional electron systems within the framework of the Tomanaga-Luttinger model. We find that Coulomb coupling has a much stronger effect on one dimensional wires than on two-dimensional layers: At zero temperature the trans-resistivity diverges, due to the formation of locked charge density waves. At temperature well above a crossover temperature $T^{*}$ the trans-resistivity follows a power law $\rho \propto T^{x}$, where the interaction-strength dependent exponent $x$ is determined by the Luttinger Liquid parameter $K_{c-}$ of the relative charge mode. At temperature below $T^{*}$ relative charge displacements are enabled by solitonic excitations, reflected by an exponential temperature dependence. The cross-over temperature $T^{*}$ depends sensitively on the wire width, inter-wire distance, Fermi wavelength and the effective Bohr radius. For wire distances $\bar{d} \gg k_{F}^{-1}$ it is exponentially suppressed with $T / E_{F} \sim \exp \left[-\bar{d} k_{F} /\left(1-K_{c-}\right)\right]$. The behavior changes drastically if each of the two wires develop spin gaps. In this case we find that the trans-resistivity vanishes at zero temperature. We discuss our results in view of possible experimental realizations in GaAs-AlGaAs semiconductor structures.
\end{abstract}

\section{INTRODUCTION}

Measurements of Coulomb drag trans-resistivity between two coupled low dimensional electronic systems are a powerful probe of scattering and correlations between electrons [1]. In a measurement of the trans-resistivity $\rho_{D}$ a current $I_{1}$ is driven in one (the "active") of the systems, while no current is allowed to flow in the other system (the "passive" system). The Coulomb interaction between electrons in the two systems transfers momentum from the active system to the passive one, where a voltage drop $V_{2}$ develops. The ratio $-V_{2} / I_{1}$ is the transresistance, which is related to the trans-resistivity by a geometric factor.

In weakly coupled two dimensional systems, at least at zero magnetic field, the trans-resistivity is usually proportional to the electron-electron momentum relaxation time, and is therefore proportional to $T^{2}$, with $T$ being the temperature. As explained by Fermi liquid theory, the $T^{2}$ behavior holds also in the presence of electronelectron interaction within each of the two coupled systems.

In one dimensional systems, which are presently realized by organic quasi-1D-metals, carbon nanotubes, edge states of quantum hall liquids, and 1D semiconducting structures, electron-electron interaction is believed to invalidate the Fermi liquid picture, and generate a different state, described approximately by the Tomanaga-Luttinger (TL) theory [2,3] (for reviews see e.g. [4] [7]). Since electronic correlations in this state are stronger than in a Fermi liquid, it is interesting to exam- ine the Coulomb drag trans-resistivity between two such systems.

In this paper we study theoretically Coulomb drag between two identical parallel one dimensional wires at close proximity. For perfectly clean wires, as assumed here, the current flowing in the active wire generates voltages on the two wires, which, due to Galilean invariance, are equal in magnitude and opposite in sign. The transresistivity $\rho_{D} \equiv-\frac{V_{2}}{I_{1} L}$ (with $L$ the length of each wire) is then also the intrinsic resistivity (not including the contact resistance) of the active wire. Thus, we occasionally refer to $\rho_{D}$ as the "resistivity". Note, however, that this resistivity does not influence a symmetric flow of current in the two wires.

Drag between 1D electron systems was considered earlier by several authors. Hu and Flensberg [8] and more recently Raichev and Vasilopoulos [9] investigated the problem in the absence of electron correlations (apart from screening effects) within Fermi liquid theory. Tanatar [10] studied the same problem in the presence of disorder, and Coulomb drag of Luttinger liquids with a point-like interaction region was considered by Flensberg [11], and Komnik and Egger [12]. In a recent work by Nazarov and Averin 13] 1D systems of spinless electrons are treated as independent Luttinger liquids with coupling limited to inter-wire backscattering ( $\Delta k \approx 2 k_{F}$, where $k_{F}$ is the Fermi wavevector in the two wires). The present work treats both intra- and interwire electron-electron interaction on equal footing. We find that although drag takes place primarily through $2 k_{F}$ scattering, the small momentum component of the

*present address: Universität zu Köln, Institut für Theoretische Physik, Zülpicher Str. 77, D-50937 Köln, Germany. 
inter-wire interaction and spin-density interactions affect it in a crucial way. The problem under consideration is also closely related to the problem of a coupled double (or $N-$ )chain 14, 15. In case of the spin-full problem results of Lee et al. "14 are useful.

The paper is organized as follows: In section II we define the problem and present the main results and the physical picture. Section III deals with two wires of spinless electrons (throughout the paper we use "wire" as synonym for "1D electron system"). After formulating this problem in subsection III B, we analyze it by means of a renormalization group in III C. We then discuss the high temperature regime (IIID), the crossover temperature (IIIE), and the low temperature regime (III F). In this analysis we employ the method developed recently by Nazarov and Averin [13], and earlier results on impurity pinned charge density waves (CDWs) [18, 19]. In section IV we address a double-wire system of spin unpolarized electrons. We write the Hamiltonian and renormalization group equation in subsection IV A, analyze the fixed points in IV B, deal with weak interactions in IV O, and extend the discussion beyond that limit in subsection IV D. We then discuss the high and low temperature limits of the drag in this case in subsection IVE. In section $\mathrm{V}$ we estimate experimental values of the relevant parameters for semiconducting wires [20,21]. section VI concludes with a summary. Some technical details are put into the appendices. In particular, in appendix B we examine the relation between the Nazarov-Averin method and earlier weak coupling calculations of Coulomb drag 25.

\section{REVIEW OF THE MAIN RESULTS}

We consider two identical wires of diameter $d$, separated by a distance $\bar{d}$. We denote by $k_{F}$ the Fermi wave vector in each wire, by $v_{F}$ the Fermi velocity in each wire. The strength of the Coulomb interaction is characterized by $r_{s}=r / a_{B}$, with $r$ the mean (intra-wire) electron distance and $a_{B}$ the effective Bohr radius. The length of the two wires is $L$.

We first consider two wires of spin-less electrons. Experimentally, this system may be realized by applying a magnetic field parallel to the wires, which would polarize the electrons' spins without affecting their orbital motion. This system is closely related to a single Luttinger liquid with a spin-degree of freedom, when the two spinprojections are identified with electrons in the two wires. Therefore, results obtained previously on the effect of backscattering in such systems [16,17] can be used.

We find that, for infinitely long wires, Coulomb coupling always leads to a diverging resistivity $\rho_{D}$ as temperature goes to zero. The physical picture behind this effect is that at sufficiently low temperature the electrons in both wires form two inter-locked CDWs. Then a relative charge displacement can be created only by overcoming a potential barrier. At zero temperature this cannot be done by an infinitesimal electric field, and leads to a non-linear trans-resistance. At finite $T$, below a crossover temperature $T^{*}$ (discussed below), the trans-resistance satisfies,

$$
\rho(T) \sim \rho_{0, T} \exp \left(E_{s} / T\right)
$$

with $E_{s} \sim T^{*}$ defined below.

For short wires a qualitative different behavior appears. Here, from time to time the CDW in the active wire slips as a whole relative to the CDW in the other wire. These instantaneous slips are a result of either thermal fluctuations or tunneling events. The latter leads to a non-diverging resistance at zero temperature, which is exponential in $L$.

At temperatures well above $T^{*}$ the previous picture of inter-locked CDWs is no longer valid. In this case it is more appropriate to think of independent electrons in the active wire, which suffer from backscattering at the $2 k_{F}$ component of the potential generated by density fluctuations in the passive wire. A perturbative calculation yields in this case a resistivity

$$
\rho(T)=\rho_{0} \lambda^{2}\left(\frac{T}{E_{0}}\right)^{x}, \quad x=4 K_{c-}-3,
$$

exhibiting a characteristic power law dependence on $T$. The coefficient $\rho_{0}$ is of order $h k_{F} / e^{2}, \lambda$ denotes the dimensionless inter-wire backscattering potential, and $E_{0}$ is of order the Fermi energy. The parameter $K_{c-}$ is the TL parameter of the relative charge density sector $(c-)$. It is determined by the difference of the small momentum intra- and inter-wire couplings, and not by the intra-wire small momentum coupling, as assumed in 13. With vanishing small-momentum interaction, $K_{c-}$ approaches unity, and $\rho(T)$ takes the linear temperature dependence of the drag resistance of independent $1 \mathrm{D}$ electrons [8]. In the presence of Coulomb interactions $K_{c-}$ may be either larger or smaller than 1 , depending on the inter-wire distance.

The crossover temperature $T^{*}$ is a complicated function of four length scales: the wire separation $\bar{d}$, wire width $d$, effective Bohr radius $a_{B}$, and the mean (intrawire) electron distance $r=\pi / k_{F}$. The first, $\bar{d}$, controls $\bar{\lambda}$, the strength of the $2 k_{F}$ component of the inter-wire Coulomb interaction. For widely separated wires $\bar{d} \gg r$, this component is exponentially small, $\bar{\lambda} \propto \exp -2 k_{F} \bar{d}$ and consequently,

$$
T^{*} \sim E_{0} \exp \left(-\frac{k_{F} \bar{d}}{1-K_{c-}}\right) .
$$

As $\bar{d} / r \rightarrow \infty$, then, $T^{*} \rightarrow 0$ and the trans-resistivity follows Eq. (11) in all practically relevant temperatures and length scales. The general trends are shown in table fi. 
The maximum values of $T^{*}$ that can be expected in realistic experimental set-ups are of order $T^{*} \sim 0.01 \times E_{0}$. In case of small wire separation $\bar{d} \ll r$ the crossover temperature $T^{*}$ is exponentially suppressed according to

$$
T^{*} \sim E_{0} \exp \left(-\frac{\pi^{3}}{r_{s}} \frac{c\left(k_{F}\right)}{k_{F} \bar{d}}\right),
$$

where $c\left(k_{F}\right)$ is of order one and only logarithmically depends on $k_{F}$.

For the spin-full case the results are similar, as long as the spin sectors are not unstable towards a formation of an energy gap in their spectrum (spin gap). The resistivity diverges at zero temperature, and scales with temperature with an exponent

$$
x=2 K_{c-}-1
$$

in the high temperature regime. A comparison with the previous exponent reveals that fluctuations in the neutral spin sector moderate the effect of the charged modes.

The behavior changes drastically if the single wires develop spin gaps. In this case we find that the transresistivity vanishes at zero temperature.

\section{SPIN-LESS DOUBLE WIRE}

\section{A. Notation}

We use the following notations:

$a_{r w}^{\dagger}(k), a_{r w}(k)$ : creation and annihilation operator of a right $(r=+)$ or left $(r=-)$ moving fermion of momentum $k$. The second index refers to the active $(w=+)$ and passive $(w=-)$ wire.

$\psi_{r w}^{(\dagger)}(x)=L^{-1 / 2} \sum_{k} e^{i k x} a_{r w}^{(\dagger)}(k) \quad:$ fermionic operators in real space representation.

$\rho_{r w}$ : density of right/left moving fermions in wire $w$.

$n_{w}=\rho_{-, w}+\rho_{+, w}:$ charge density of wire $w$.

$n_{c \pm}=n_{-}+n_{+}:$absolute (or symmetric, $c+$ ) and relative (or antisymmetric, $c-$ ) charge density of the double wire system.

In general, the indices $c+$ and $c-$ refer to quantities of the absolute and relative charge mode, respectively. We also use this convention for bosonic field, introduced in the next subsection.

The notation we use for the coupling constants follows that of Voit's review [4. We use $g_{i}$ to denote intra-wire couplings, and $\bar{g}_{i}$ to denote inter-wire couplings. The subscript $i=1$ denotes $2 k_{F}$ scattering, $i=2,4$ denote small momentum scattering.

\section{B. The Hamiltonian in fermionic and bosonic representation}

In this section we consider two one dimensional wires of spin polarized electrons with equal densities. If the wire index is viewed as a z-component of an "iso-spin", the Hamiltonian of the problem is that of an iso-spin- $\frac{1}{2}$ $1 \mathrm{D}$ system (but with interactions that are not $S U(2)$ symmetric), and results obtained previously on the effect of backscattering in such systems 16,17 can be used. The kinetic energy part of the Hamiltonian states 《4,

$$
H_{0}=v_{F} \sum_{r w k} r k a_{r w}^{\dagger}(k) a_{r w}(k)=\frac{\pi v_{F}}{L} \sum_{q r w} \rho_{r w}(q) \rho_{r w}(-q)
$$

The small-momentum transfer or forward scattering part of the electron-electron interaction is given by [4

$$
\begin{aligned}
H_{f}= & \frac{1}{L} \sum_{w w^{\prime} q}\left[\delta_{w, w^{\prime}} g_{2}+\delta_{w,-w^{\prime}} \bar{g}_{2}\right] \rho_{+w}(q) \rho_{-w^{\prime}}(-q) \\
& +\frac{1}{2 L} \sum_{r w w^{\prime} q}\left[\delta_{w, w^{\prime}} g_{4}+\delta_{w,-w^{\prime}} \bar{g}_{4}\right] \rho_{r w}(q) \rho_{r w^{\prime}}(-q)
\end{aligned}
$$

and backscattering processes are described by [4

$$
\begin{gathered}
H_{b}=\sum_{w w^{\prime}} \int d x \psi_{+w}^{\dagger}(x) \psi_{-w^{\prime}}^{\dagger}(x) \psi_{+w^{\prime}}(x) \psi_{-w}(x) \times \\
{\left[\delta_{w, w^{\prime}} g_{1}+\delta_{w,-w^{\prime}} \bar{g}_{1}\right] .}
\end{gathered}
$$

It is convenient to switch to a standard boson representation by introducing bosonic fields $\phi_{w}(x)=$ $-i \pi L^{-1} \sum_{q} q^{-1} e^{-i q x-\alpha|q| / 2}\left(\rho_{+w}(q)+\rho_{-w}(q)\right)$ with their conjugates $\Pi_{w}(x)=L^{-1} \sum_{q} e^{-i q x-\alpha|q| / 2}\left(\rho_{+w}(q)-\right.$ $\left.\rho_{-w}(q)\right)[7]$. Throughout the paper we interpret the length $\alpha$ as the inverse Fermi wavevector $2 \pi / k_{F}$. Physically, the field $\phi_{w}(x)$ denotes the displacement of electrons in wire $w$, normalized in such a way that density fluctuations $\delta n_{w}(x)$ and current $I_{w}(x)$ are given by $\partial_{x} \phi_{w}(x)=-\pi \delta n_{w}(x)$ and $\partial_{t} \phi_{w}(x)=\pi I_{w}(x)$. The relation to the fermions $\psi_{r w}$ is established by the Luther-Peschel transformation formula, $\psi_{r w}(x)=$ $(2 \pi \alpha)^{-1 / 2} \exp \left[\operatorname{ir}\left(k_{F} x-\phi_{w}(x)\right)+\theta_{w}(x)\right]$, where $\theta(x)=$ $\pi \int_{x_{0}}^{x} d x^{\prime} \Pi_{w}\left(x^{\prime}\right)$ [7].

The total Hamiltonian $H=H_{0}+H_{f}+H_{b}$ separates into two decoupled parts, one describing absolute (symmetric) current and density, and one describing relative (antisymmetric) current and density. The decoupling is obtained by means of the transformation, $\phi_{c \pm}=2^{-1 / 2}\left(\phi_{+} \pm \phi_{-}\right), \Pi_{c \pm}=2^{-1 / 2}\left(\Pi_{+} \pm \Pi_{-}\right)$[16]. In bosonic representation the two parts are [4] 


$$
\begin{gathered}
H_{c+}=\frac{u_{c+}}{2 \pi} \int d x\left(K_{c+} \pi^{2} \Pi_{c+}^{2}+\frac{1}{K_{c+}}\left(\partial_{x} \phi_{c+}\right)^{2}\right), \\
H_{c-}=\frac{u_{c-}}{2 \pi} \int d x\left(K_{c-} \pi^{2} \Pi_{c-}^{2}+\frac{1}{K_{c-}}\left(\partial_{x} \phi_{c-}\right)^{2}\right) \\
\quad+\frac{2 \bar{g}_{1}}{(2 \pi \alpha)^{2}} \int d x \cos \left(\sqrt{8} \phi_{c-}\right), \\
K_{c \pm}=\sqrt{\frac{1+U_{c \pm}}{1-U_{c \pm}}}, \\
U_{c+}=\frac{1}{2 \pi v_{c+}}\left(-g_{2}-\bar{g}_{2}+g_{1}\right), \\
U_{c-}=\frac{1}{2 \pi v_{c-}}\left(-g_{2}+\bar{g}_{2}+g_{1}\right), \\
v_{c \pm}=v_{F}+\left(g_{4} \pm \bar{g}_{4}\right) / 2 \pi \\
u_{c \pm}=v_{c \pm}\left(1-U_{c \pm}^{2}\right)^{1 / 2} .
\end{gathered}
$$

(The signs in the definition of the small momentum couplings $U_{c \pm}$ are chosen according to the conventions in [17].) The fields $\phi_{c \pm}$ describe fluctuations in the absolute and relative density via $\partial_{x} \phi_{c \pm}=-2^{-\frac{1}{2}} \pi \delta n_{c \pm}$. Accordingly, the relation to currents are $\partial_{t} \phi_{c \pm}=2^{-\frac{1}{2}} \pi I_{ \pm}$.

The current in a drag experiment is a superposition of a symmetric and an anti-symmetric current. A symmetric current flows without resistivity, due to Galilean invariance. Thus, the resistance results from the antisymmetric part only, and is determined by the relative charge sector $H_{c-}$ only. Formally this is manifested in the invariance of $H_{c+}$ to spatially homogeneous charge displacements $\phi_{c \pm}(x) \rightarrow \phi_{c \pm}(x)+\varphi_{c \pm}$, an invariance which is absent in the backscattering potential $\left(\propto \cos \sqrt{8} \phi_{c-}\right)$ in $H_{c-}$. Consequently, we confine ourselves to the sineGordon type Hamiltonian $H_{c--}$.

The Hamiltonian $H_{c-}$ has two parameters, $K_{c-}$ (which may be expressed in terms of $U_{c-}$, see Eq. (6)) and $\bar{g}_{1}$. Our results are all independent of the sign of $\bar{g}_{1}$, which we take below to be positive. For a single wire with electrons of two spin directions (namely, for $S U(2)$ symmetric interaction, $g_{2}=\bar{g}_{2}$ ) the sign of $U_{c-}$ is determined by the sign of $g_{1}$, i.e., by whether the interaction is repulsive or attractive. Here this only holds for two wires which are very close to one another. For larger inter-wire distances $U_{c-}$ can become negative also for repulsive interaction, in particular Coulomb interaction: Then, $\bar{g}_{2}-g_{2}=-\frac{2 e^{2}}{\epsilon} \ln \bar{d} / d$, while the parameter $g_{1}$ is independent of $\bar{d}$. Thus, for large inter-wire distance $\bar{d}$, the parameter $U_{c-}<0$.

Below we confine ourselves to repulsive interaction, and discuss the case of wires at close proximity and that of well separated wires.

\section{Renormalization group analysis}

In this subsection we analyze the backscattering term of the Sine-Gordon Hamiltonian by means of a renormal- ization group (RG) analysis, and show that if the bare interactions are weak, and the electron-electron interaction potential decays with distance, the drag resistivity diverges at zero temperature.

Let us first recall the main elements of an RG treatment for $H_{c-}$ (see e.g. 四). For small backscattering couplings, $\bar{\lambda} \equiv \bar{g}_{1} / 2 \pi u_{c-} \ll 1$, the RG equations are of the Kosterlitz-Thouless type (here we denote $K \equiv K_{c-}$ )

$$
\frac{d \bar{\lambda}}{\mathrm{d} x}=(2-2 K) \bar{\lambda}, \quad \frac{d K}{\mathrm{~d} x}=-2 \bar{\lambda}^{2} K^{2},
$$

where the parameter $x=\ln l / \alpha$ is the logarithm of the renormalized momentum cut-off $l^{-1}$. The RG procedure starts with the bare couplings $\bar{\lambda}_{0}, K_{0}$ at an initial momentum cut-off $l_{0}^{-1}$ of order of $\alpha^{-1}$ and ends with renormalized couplings at a final cut-off $l_{1}^{-1}=\max \left\{L^{-1}, T / u_{c-}\right\}$. One method (due to Jose et al. 22 to derive the RG equations is to expand the scale invariant correlation function $\left\langle e^{2 i \phi_{c-}\left(x_{1}, \tau_{1}\right)} e^{-2 i \phi_{c-}\left(x_{2}, \tau_{2}\right)}\right\rangle$ in powers of the coupling $\bar{\lambda}$ and to integrate out the large momentum degrees of freedom. After re-exponentiating the result one can then read off the RG equations (for details see [22] and also [23]). Within each $R G$ step only the backscattering interaction $\bar{g}_{1}$ is treated perturbatively, whereas the small momentum interaction parameters $g_{2}-g_{1}, g_{4}, \bar{g}_{2}, \bar{g}_{4}$, which determine the parameter $K$, are treated exactly. A different method, which leads to the same RG equations (11) but may provide additional insights, is by means of mapping the Sine-Gordon Hamiltonian onto the two-dimensional Coulomb gas problem 117.

The integral curves $\bar{\lambda}(K)$ of the RG-flow (11) shown in Fig. (1) obey the differential equation

$$
\frac{\mathrm{d} \bar{\lambda}}{\mathrm{d} K}=\frac{K-1}{K^{2} \bar{\lambda}}
$$

and are of the form

$$
\bar{\lambda}(K)=\sqrt{2}\left(\frac{1}{K}+\ln \left(K / K_{0}\right)-\frac{1}{K_{0}}+\frac{\bar{\lambda}_{0}^{2}}{2}\right)^{1 / 2} .
$$

There are two types of stable fixed points to Eqs. (11). Fixed points of the first type are characterized by $\bar{\lambda}=0, K>1$, i.e., by zero drag at $T=0$. The basin of attraction of these fixed points is the area below the separatrix $\bar{\lambda}_{s}(K)=\sqrt{2}\left(\frac{1-K}{K}+\log K\right)^{1 / 2}$. Systems with bare couplings $\bar{\lambda}_{0}, K_{0}$ inside this area scale towards weaker backscattering coupling $\bar{\lambda}$ when temperature decreases. Below we show that no realistic set of interaction parameters falls under this category.

When the bare couplings are outside this region (i.e. $K_{0}<1$ or $\bar{\lambda}_{0}>\bar{\lambda}_{s}\left(K_{0}\right)$ ) renormalization to lower temperature drives the system into the strong coupling stable fixed point, where $\bar{\lambda} \rightarrow \infty$ and $K \rightarrow 0$. In this case backscattering becomes dominant at sufficiently 
low temperatures and freezes the phase $\phi_{c-}$ to a minimum position. Translated to the double-wire system this means that the charges adjust their relative displacement $\sqrt{2} \phi_{c-}=\phi_{+}-\phi_{-}$in such a way that the $2 k_{F}$ inter-wire potential is minimal, i.e. the system forms two interlocked CDWs.

Under these conditions the drag is very strong, as pointed out by Nazarov and Averin [13]. The system's resistivity to a flow of unequal currents in the two wires becomes infinite, in the limit of zero temperature and infinite length. We elaborate on this subject in section (III F).

It is instructive to express the condition for weak and strong coupling in terms of the bare interaction parameters $g_{i}, \bar{g}_{i}$. For reasonably weak interaction, $K \lesssim 1.6$, the separatrix $\bar{\lambda}_{s}(K)$ is well approximated by $\bar{\lambda}_{s}(K) \approx$ $K-1 \approx U_{c-}$. Within this approximation the requirement for strong coupling, $\bar{\lambda}_{0}>\bar{\lambda}_{s}\left(K_{0}\right)$, states

$$
\bar{\lambda}_{0}>U_{c-}^{0} .
$$

which is equivalent to

$$
\left(g_{2}-g_{1}\right)>\left(\bar{g}_{2}-\bar{g}_{1}\right)
$$

Rather than the absolute strength of the inter-wire interaction couplings, it is their difference $\bar{g}_{2}-\bar{g}_{1}$ in comparison to $g_{2}-g_{1}$ which determines the zero temperature fixed point of the system. Taking $g_{i}$ and $\bar{g}_{i}$ as the Fourier components of intra- and inter-wire interaction $V(x)$ and $\bar{V}(x)$ at $q_{1}=2 k_{F}$ and $q_{2}=1 / L \rightarrow 0$, the condition (13) becomes

$$
\int d x\left(1-\cos q_{1} x\right)[V(x)-\bar{V}(x)]>0 .
$$

The first factor is non-negative. Then, a sufficient condition for the l.h.s. to be positive is obviously $V(x)>\bar{V}(x)$ for all $x$, which is fulfilled by all monotonously decaying repulsive interactions potentials, in particular the Coulomb potential. Therefore, a Coulomb coupled double wire system of spin-less electrons should scale towards strong coupling, which implies a diverging zero temperature drag in infinitely long wires.

There are two types of initial values $\bar{\lambda}_{0}, K_{0}$ which flow to the strong coupling fixed point. The first is defined by $K_{0}<1$. As seen in Eqs. (11), for this case $\bar{\lambda}$ varies monotonously as the temperature decreases. The second type is defined by $K_{0}>1$ and $\bar{\lambda}_{0}>\bar{\lambda}_{s}\left(K_{0}\right)$. For this type, $\bar{\lambda}$ does not varies monotonously. For relatively high temperature, $\bar{\lambda}$ decreases. At the temperature at which $K=1 \bar{\lambda}$ starts increasing towards the strong coupling fixed point. As explained in the previous subsection, well-separated wires $(\bar{d} \rightarrow \infty)$ fall under the first category, while wires at very close proximity fall under the second. Assuming the bare interaction parameters $g_{i}, \bar{g}_{i}$ to be small, for both types of initial conditions we may separate between a weak coupling, high temperature, regime, where perturbation theory calculations can be carried out, and a low temperature, strong coupling regime. In the next subsections we calculate the drag resistivity for both regimes, and identify the temperature scale that separates the two.

\section{The high temperature regime}

We begin with the weakly coupled regime, in which we employ a method devised by Nazarov and Averin [13]. In the limit of linear response $(I \rightarrow 0)$ this approach of calculating the drag resistivity is similar to the memoryfunction formalism of Zheng and MacDonald [25], as it is shown in appendix B. The main difference is that in the present calculation only the backscattering component of the inter-wire interaction is treated perturbatively, while the small-momentum part is treated exactly.

We consider a four-probe measurement with voltage probes at positions $x_{0}$ and $x_{0}+a$ on both wires (let $\left.u_{c-} / T \ll a \ll L\right)$, and calculate the voltage drop $e V_{w}=\left\langle\mu_{w}\left(x_{0}\right)-\mu_{w}\left(x_{0}+a\right)\right\rangle_{I}$ along wire $w$ when a current $I$ is driven only through the active wire $(+)$. Using the relation $\delta e V_{w}=\kappa^{-1} \delta n_{w}$, where $\kappa^{-1}=\partial \mu_{w} / \partial n_{w}$ is the inverse compressibility, we obtain,

$$
e V_{w}=\frac{1}{\kappa}\left\langle\delta n_{w}(x+a)-\delta n_{w}(x)\right\rangle_{I}=-\frac{1}{\kappa} \int_{x_{0}}^{x_{0}+a}\left\langle\partial_{x} n_{w}\right\rangle_{I}
$$

Due to translational invariance,

$$
\frac{e V_{w}}{a}=\frac{1}{\kappa}\left\langle\partial_{x} n_{w}\right\rangle_{I}=-\frac{\pi}{\sqrt{2} \kappa}\left\langle\partial_{x}^{2} \phi_{c+}+w \partial_{x}^{2} \phi_{c-}\right\rangle_{I} .
$$

The thermo-dynamical averaging $\langle\ldots\rangle_{I}$ has to be restricted to states satisfying $\left\langle I_{+}\right\rangle=I$ and $\left\langle I_{-}\right\rangle=0$. Equivalently, but technically more convenient, one can use an ensemble of current-less states, and then perform a Galilean-transformation of the active wire such that a net current $I$ results. In terms of the displacement fields $\phi_{s}$ this means that $\phi_{+}$acquires a component growing linearly in time,

$$
\phi_{+}(x, t) \rightarrow \phi_{+}(x, t)+\Omega t,
$$

or, translated into absolute and relative fields,

$$
\phi_{c \pm} \rightarrow \phi_{c \pm}+\frac{\Omega t}{\sqrt{2}}
$$

The frequency $\Omega$ is related to the current by $\Omega=\pi I / e$ 24 .

As expected, the transformation (16) does not alter the absolute sector $H_{c+}$, and no symmetric voltage is induced by the current. It does affect the relative Hamiltonian $H_{c-}$ via the backscattering interaction, which becomes 


$$
H_{\text {int }}=\bar{\lambda} E_{0} \int \frac{d x}{\pi \alpha} \cos \left(\sqrt{8} \phi_{c-}+2 \Omega t\right),
$$

and gives rise to a finite drag-voltage

$$
\frac{e V_{w}}{a}=w \frac{\pi \kappa}{\sqrt{2}}\left\langle\partial_{x}^{2} \phi_{c-}\right\rangle_{H_{\text {int }}} .
$$

For the following calculation it is advantageous 13] to make use of the equation of motion, $\partial_{t}^{2} \phi_{c-}=$ $-\left[H_{c-},\left[H_{c-}, \phi_{c-}\right]\right]$, from which follows that under stationary conditions $\left(\left\langle\partial_{t}^{2} \phi_{c-}\right\rangle=0\right)$

$$
\begin{aligned}
\left\langle\partial_{x}^{2} \phi_{c-}\right\rangle & =-\frac{K}{u}\left\langle\frac{\delta H_{\text {int }}}{\delta \phi}\right\rangle \\
& =\frac{\sqrt{8} K}{\alpha^{2}} \bar{\lambda}\langle\sin (\sqrt{8} \phi+2 \Omega t)\rangle,
\end{aligned}
$$

and then to perform a perturbative expansion of the r.h.s in the backscattering coupling $\bar{\lambda}$ with respect to $H_{0}=H_{c-}-H_{\text {int }}$.

By standard methods we obtain in lowest nonvanishing order the resistivity

$$
\left.\rho_{w} \equiv \frac{\partial}{\partial I} \frac{V_{w}}{l}\right|_{I=0}=w \rho_{0} \bar{\lambda}^{2}\left(\frac{T}{E_{0}}\right)^{4 K-3}
$$

where $\rho_{0} \sim h / e^{2} \alpha$. Higher order terms $\rho^{(n)} \propto \bar{\lambda}^{2 n}$ scale with temperature as

$$
\rho^{(n)} \propto T^{\delta_{n}}, \quad \delta_{n}=(4 K-4) n+1 .
$$

Terms with odd powers of $\bar{g}_{1}$ vanish.

The temperature scaling law $\rho \propto T^{4 K-3}$ can be also derived from the RG treatment in the following way: In the absence of intra-wire interaction a simple calculation yields

$$
\rho(T) \sim \rho_{0} \bar{\lambda}_{0}^{2} T / E_{0}
$$

The effect of intra-wire scattering can be accounted for by using a renormalized backscattering coupling constant $\bar{\lambda}=\bar{\lambda}(T)$. One finds from the RG equations (11) that in the weak coupling limit $\bar{\lambda}(T)=\bar{\lambda}_{0}\left(T / E_{0}\right)^{2 k-2}$ (see appendix A, Eq. (A1)), which inserted into the previous equation indeed gives $\rho(T) \propto \bar{\lambda}_{0}^{2}\left(T / E_{0}\right)^{4 K-3}$.

\section{E. The crossover temperature}

In this section we estimate the cross-over temperature below which the perturbative calculation of the previous section ceases to hold, and inter-locking of the two charge density waves in the two wires becomes relevant. The cross-over temperature is that in which the coupling constant $\bar{\lambda}$ becomes of order 1 .
For initial conditions $\bar{\lambda}_{0} \ll 1$ and $K_{0}<1$, corresponding to well separated wires, the solution to the RG equations can be approximated by $\bar{\lambda}(T) \approx \bar{\lambda}_{0}\left(\frac{E_{0}}{T}\right)^{2-2 K_{0}}$ and $K \approx K_{0}$. The cross-over is then

$$
T^{*} \sim E_{0} \bar{\lambda}_{0}^{1 /\left(2-2 K_{0}\right)}
$$

In appendix $\mathrm{A}$ it is shown that for $\bar{d} \gg d, k_{F}$ and for the Coulomb interaction, the coupling constant $\bar{\lambda}_{0} \sim$ $\exp -k_{F} \bar{d}$, and thus $T^{*}$ is exponentially suppressed. More specifically, we find in this case

$$
T^{*} \sim E_{0} \exp \left(-\frac{k_{F} \bar{d}}{1-K_{0}}\right),
$$

Note that with vanishing interaction strength, $1-K_{c-} \approx$ $-U_{c-} \approx r_{s}\left(\ln k_{F} \bar{d}+\gamma\right) \pi^{-2}$ (see section $\mathrm{V}$ ), and hence $T^{*} \propto \exp \left(-\frac{k_{F} \bar{d}}{r_{s} \ln k_{F} d}\right)$, where the parameter $r_{s} \equiv r / a_{B}=$ $\pi e^{2} / v_{F} \epsilon$ describes the interaction strength.

For initial conditions of the type $K_{0} \gtrsim 1$ and $\bar{\lambda}_{0}>$ $\bar{\lambda}_{s}(K)$, corresponding to wires at very close proximity, the RG equations can again be solved approximately (see Appendix (A) for details), and we find,

$$
T^{*} \sim E_{0} \exp \left(-\frac{\pi^{3}}{r_{s}} \frac{c\left(k_{F}\right)}{k_{F} \bar{d}}\right),
$$

where $c\left(k_{F}\right)$ is of order one and only logarithmically depends on $k_{F}$. Thus, when $1 / k_{F} \bar{d} \gg 1$, the temperature $T^{*}$ is again exponentially small. At first sight, this might be surprising. However, it becomes understandable by observing that for $\bar{d} \ll r$ intra and inter wire coupling are almost equal. Hence the problem becomes close to that of a single wire with electrons of two spin states, (where $g_{i}=\bar{g}_{i}$ ), which is known to renormalize to the marginal weak coupling fixed point.

\section{F. Low temperature limit}

In the strong coupling limit, with $\bar{\lambda} \gtrsim 1$, and $K \ll 1$, the Sine-Gordon Hamiltonian (5) describes two interlocked charge density waves, and carries strong resemblance to the problem of a pinned charge density wave, which has been studied quite extensively in the 1970s (we refer in particular to the work of Rice et al. [18] and Maki [19]).

Classically, for large $\bar{\lambda}$ the Hamiltonian $H_{c-}$ has an infinite number of ground states with field configurations $\phi(x) \equiv \phi_{N}=\pi(2 N+1) / \sqrt{8}$. They are separated by a large energy barrier, such that at sufficiently low temperatures the field fluctuates around one of the ground states $\phi_{N}$. These fluctuations do not carry an anti-symmetric current. Rather, it is a transition from a ground state $\phi_{N}$ to its neighbor $\phi_{N \pm 1}$ which corresponds to an electron transfer through the active wire from the left end 
to the right end or vice versa. These transitions are carried out either by a soliton moving along the wire, or by a soliton-antisoliton pair which is created in the wire and is dissociated by an electric field. The width $W$ and energy $E_{s}$ of a classical soliton are 26]

$$
W \sim \frac{\alpha}{\sqrt{K \bar{\lambda}}}, \quad E_{s}=\sqrt{\frac{8 \bar{\lambda}}{\pi^{2} K}} E_{0} .
$$

We expect that the transport properties of short wires with $L \ll W$ are different from that of long wires, where $L \gg W$, and therefore consider both regimes separately.

In long wires electronic current is carried by means of soliton-antisoliton pairs. This mechanism was studied by Rice et al. [18] and Maki [19] within a semi-classical approximation. The semi-classical regime is that in which the classical soliton energy $E_{s}$ is much larger than the zero point energy of the fluctuations around it. In terms of our parameters this regime is defined by $K \ll 1$. As found by Rice et al. [18], in this regime thermal creation of soliton-antisoliton pairs leads to a resistivity

$$
\rho=\frac{h}{16 \pi e^{2} l} \sqrt{\frac{E_{s} T}{2 \bar{\lambda} K E_{0}^{2}}} e^{E_{s} / T},
$$

where $l$, the mean free path of (anti)solitons, is a phenomenological parameter.

When $K \ll 1$, the soliton energy is renormalized according to 27

$$
E_{s}^{r}=E_{s}\left(\frac{E_{s}}{E_{0}}\right)^{\frac{K}{1-K}} .
$$

Eqs. 24 and (25) define a crossover temperature $T^{*} \equiv$ $E_{s}^{r}$ separating the low and high temperature regimes. As one may expect, this temperature is approximately equal to the one obtained in Subsec. IIIF.

The low temperature regime is characterized by a highly non linear drag, taking place at exceedingly low temperatures, where soliton-antisoliton pairs are generated by quantum tunneling, rather than thermal activation. In this regime, as found by Maki,

$$
\rho=\frac{h}{e^{2}} \frac{E_{s}}{16 \pi^{2} u_{c-}} e^{\epsilon_{0} / \epsilon},
$$

where $\epsilon_{0}=E_{s}^{2} / 2 e u_{c-}, \epsilon=V / L$, and $V$ is the voltage difference between the two ends of the wires. The crossover temperature, at which thermally activated behavior changes to a tunneling dominated one, can be determined by equating the exponentials, which yields

$$
T_{c o} \sim 2 \sqrt{\frac{K}{\bar{\lambda}}} \frac{\alpha}{L} e V .
$$

For short wires, where the soliton width $W$ is large compared to the wire length $L$, the fields $\Pi, \phi$ may be approximated as constant in space, leading to an Hamiltonian

$$
\begin{aligned}
H & =\frac{u_{c-} L}{2 \pi}\left[\pi^{2} K_{c-} \Pi^{2}+\frac{2 \bar{\lambda}}{\alpha^{2}} \cos \sqrt{8} \phi_{c-}\right] \\
& \equiv \frac{\omega^{2}}{E_{b}} p^{2}+\frac{E_{b}}{2} \cos \varphi .
\end{aligned}
$$

Here we introduced a frequency $\omega^{2}=8 \bar{\lambda} K E_{0}^{2}$, a barrier energy $E_{b}=\frac{2}{\pi} \bar{\lambda} \frac{L}{\alpha} E_{0}$, and canonical conjugated variables $\varphi=\sqrt{8} \phi, p=L \Pi / \sqrt{8}$. While this Hamiltonian resembles that of a pendulum, it differs from it in one important aspect: in the present case the states $\varphi$ and $\varphi \pm 2 \pi$ are distinct physical states, since a shift of the phase by $2 \pi$ corresponds to a transfer of one electron between the reservoirs the active wire is coupled to. Since different minima of the potential energy correspond to distinct states of the reservoirs, it is unlikely that the field $\phi$ can be in a superposition of several such minima. We therefore assume that the field is in one of the minima, and transport takes place by tunneling or thermal hopping between adjacent minima.

A chemical potential difference $\delta \mu$ between the reservoirs at the ends of the active wire adds a linear potential to the Hamiltonian (27)

$$
V(q)=\frac{\delta \mu}{2 \pi} \varphi .
$$

To determine the current induced by $\delta \mu$ we calculate the rates $\Gamma_{l / r}$ by which the phase $\varphi$ hops between minima by thermal activation or tunneling. Then

$$
I=e\left(\Gamma_{r}-\Gamma_{l}\right)
$$

Thermal activated hopping dominates at temperatures $T \gg \omega$ with a rate 28

$$
\Gamma_{l / r} \approx \frac{\omega}{2 \pi} e^{-E_{b} / T} e^{\mp \delta \mu / 2 T}
$$

At low temperatures $T \ll \omega$ the main contribution comes again from tunneling processes with rate

$$
\Gamma_{l / r}^{\prime} \sim \omega e^{-S_{l / r}(\delta \mu)}
$$

For $\delta \mu \ll E_{b}$ the action can be expressed as

$$
\begin{aligned}
S_{l / r}(\delta \mu) & =\frac{E_{b}}{\sqrt{2} \omega} \int_{\sigma}^{2 \pi-\sigma} d \varphi \sqrt{1-\cos \varphi \pm \frac{\delta \mu}{\pi E_{b}}} \\
& \approx \frac{4 E_{b}}{\omega} \pm C \frac{\delta \mu}{\omega}
\end{aligned}
$$

where $\sigma \sim\left(K \alpha^{2} / \bar{\lambda} L^{2}\right)^{1 / 4} \ll 1$ takes care of the finite width of the well ground state. The coefficient $C$ is of order unity and only logarithmically dependent on $\sigma$.

Putting this together we end up with current-voltage relations 


$$
\begin{aligned}
& I \approx e \frac{\omega}{\pi} e^{-E_{b} / T} \sinh (\delta \mu / 2 T), \quad \text { for } \quad T \gg \omega \\
& I \sim e \omega e^{-4 E_{b} / \omega} \sinh (C \delta \mu / \omega), \quad \text { for } \quad T \ll \omega .
\end{aligned}
$$

Accordingly, the linear resistance is

$$
\begin{aligned}
& R \approx \frac{h}{e^{2}} \frac{T}{\omega} e^{E_{b} / T}, \quad \text { for } \quad T \gg \omega, \\
& R \sim \frac{h}{e^{2}} e^{4 E_{b} / \omega}, \quad \text { for } \quad T \ll \omega .
\end{aligned}
$$

These expressions are valid for wires much shorter than the soliton length $W \sim \alpha / \sqrt{K \bar{\lambda}}$ and voltages $e V \ll E_{b}$. Notice that because of $E_{b} \propto L / \alpha$ the resistance increases exponentially with length $L$.

\section{SPIN-FULL DOUBLE WIRE}

\section{A. The Hamiltonian and renormalization group equations}

We now extend our analysis to include the spin degree of freedom of electrons in the two wires. Denoting the spin degree of freedom by an index $s= \pm$ in addition to $r, w$ as above, and taking into account the $S U(2)$ symmetry of the Coulomb interaction, we have

$$
\begin{aligned}
& H_{0}=\frac{\pi v_{F}}{L} \sum_{q r w s} \rho_{r s w}(q) \rho_{r s w}(-q) \\
& H_{f}=\frac{1}{L} \sum_{q s w s^{\prime} w^{\prime}}\left[\delta_{w, w^{\prime}} g_{2}+\delta_{w,-w^{\prime}} \bar{g}_{2}\right] \rho_{+s w}(q) \rho_{-s^{\prime} w^{\prime}}(-q) \\
&+\frac{1}{2 L} \sum_{r q s w s^{\prime} w^{\prime}}\left[\delta_{w, w^{\prime}} g_{4}+\delta_{w,-w^{\prime}} \bar{g}_{4}\right] \rho_{r s w}(q) \rho_{r s^{\prime} w^{\prime}}(-q), \\
& H_{b}=\sum_{s w s^{\prime} w^{\prime}} \int d x\left[\delta_{w, w^{\prime}} g_{1}+\delta_{w,-w^{\prime}} \bar{g}_{1}\right] \times \\
& \psi_{+s w}^{\dagger}(x) \psi_{-s^{\prime} w^{\prime}}^{\dagger}(x) \psi_{+s^{\prime} w^{\prime}}(x) \psi_{-s w}(x) .
\end{aligned}
$$

Following the same procedure as in the spin-less case, we first change to bosonic field variables [7]

$$
\begin{aligned}
& \phi_{s w}(x)=-\frac{i \pi}{L} \sum_{q \neq 0} \frac{e^{-i q x-\alpha|q| / 2}}{q}\left(\rho_{+s w}(q)+\rho_{-s w}(q)\right), \\
& \Pi_{s w}(x)=\frac{1}{L} \sum_{q \neq 0} e^{-i q x-\alpha|q| / 2}\left(\rho_{+s w}(q)-\rho_{-s w}(q)\right),
\end{aligned}
$$

and then transform to new bosonic fields $\tilde{\phi}_{c / s \pm}, \tilde{\Pi}_{c / s \pm}$ corresponding to absolute $(+)$ and relative $(-)$ charge $(c)$ and spin $(s)$ density. The transformation is defined by

$$
\phi_{s w}=\frac{1}{2}\left(\tilde{\phi}_{c+}+s \tilde{\phi}_{s+}+w \tilde{\phi}_{c-}+s w \tilde{\phi}_{s-}\right),
$$

and in the same way for $\tilde{\Pi}_{c / s \pm}$. For the transformation of the backscattering Hamiltonian $H_{b}$ we use the formula

$$
\psi_{r s w}=\frac{\eta_{r s w}}{\sqrt{2 \pi \alpha}} \exp \left[i r\left(k_{F} x-\phi_{s w}\right)+i \theta_{s w}\right]
$$

where $\theta_{s w}\left(x_{0}\right)=\pi^{-1} \int_{0}^{x_{0}} \Pi_{s w} d x$ and the $\eta_{r s w}=\eta_{r s w}^{\dagger}$ are so-called Majorana fermions. They obey

$$
\left[\eta_{r s w}, \eta_{r^{\prime} s^{\prime} w^{\prime}}\right]_{+}=2 \delta_{r s w, r^{\prime} s^{\prime} w^{\prime}}
$$

and ensure that the operators given by (32) follow fermionic commutation rules [7]. We obtain the following Hamiltonian (the tilde is omitted, $j$ takes the values $j=c, s$, for charge and spin. $l$ takes the values $l= \pm$, for symmetric and anti-symmetric)

$$
\begin{gathered}
H=\sum_{j l} H_{j l}+H_{b}, \\
H_{j l}=\frac{u_{j l}}{2 \pi} \int K_{j l} \pi^{2} \Pi_{j l}^{2}+\frac{1}{K_{j l}}\left(\partial_{x} \phi_{j l}\right)^{2}, \\
K_{j l}=\left(1+U_{j l}\right)^{1 / 2}\left(1-U_{j l}\right)^{-1 / 2}, \\
U_{c+}=\frac{1}{2 \pi v_{c+}}\left(-2 g_{2}-2 \bar{g}_{2}+g_{1}\right), \\
U_{c-}=\frac{1}{2 \pi v_{c-}}\left(-2 g_{2}+2 \bar{g}_{2}+g_{1}\right), \\
U_{s \pm}=\frac{1}{2 \pi v_{s \pm}} g_{1}, \\
v_{c \pm}=v_{F}+\left(g_{4} \pm \bar{g}_{4}\right) / \pi \\
u_{s \pm}=v_{F}, \\
H_{b}=+\frac{g_{1}}{\pi^{2} \alpha^{2}} \int d x \cos 2 \phi_{s+} \cos 2 \phi_{s-} \\
-\frac{\bar{g}_{1}}{\pi^{2} \alpha^{2}} \int d x \cos 2 \phi_{c-} \cos 2 \phi_{s-} \\
-\frac{\bar{g}_{1}}{\pi^{2} \alpha^{2}} \int d x \cos 2 \phi_{c-} \cos 2 \phi_{s+} \cdot
\end{gathered}
$$

Again, the system has no resistance to a symmetric flow of current, due to Galilean invariance, and thus the charge symmetric part of the Hamiltonian $H_{c+}$ decouples from the other parts. The remaining parts are all coupled by the backscattering Hamiltonian $H_{b}$, so that the spin modes, despite their charge neutrality, do affect the role of the Coulomb interaction.

As in the spin polarized case, when the backscattering couplings $g_{1}, \bar{g}_{1}$ scale to the strong coupling fixed point at $T=0$, spin fields $\phi_{s \pm}$ and the relative charge field $\phi_{c-}$ freeze to minimum positions, which causes a divergent drag resistance at zero temperature. On the other hand, when $\bar{\lambda}$ renormalizes to zero (while $\lambda$ can still scale to stronger couplings), the drag resistance decreases with temperature and vanishes at $T=0$. (The case of a decreasing $\lambda$ and an increasing $\bar{\lambda}$ does not exist.)

The second order RG-equations for a double wire as described by the Hamiltonian above are 


$$
\begin{aligned}
\frac{d \bar{\lambda}}{d x} & =\left(2-K_{s}-K_{c}-2 \lambda\right) \bar{\lambda}, \\
\frac{d \lambda}{d x} & =\left(2-2 K_{s}\right) \lambda-2 \bar{\lambda}^{2}, \\
\frac{d K_{s}}{d x} & =-2\left(\bar{\lambda}^{2}+\lambda^{2}\right) K_{s}^{2}, \\
\frac{d K_{c}}{d x} & =-4 \bar{\lambda}^{2} K_{c}^{2}
\end{aligned}
$$

where $\bar{\lambda} \equiv-\bar{g}_{1} / 2 \pi u_{c-}, \lambda \equiv g_{1} / 2 \pi u_{s \pm}, K_{c} \equiv K_{c-}$, and $K_{s} \equiv K_{s \pm}$. These equations can be derived in the same manner as those in the spin polarized case by assuming scale invariance of the correlation functions $\left\langle e^{2 i \phi_{j l}\left(x_{1}, \tau_{1}\right)} e^{-2 i \phi_{j l}\left(x_{2}, \tau_{2}\right)}\right\rangle$ 22,23. They are valid for arbitrary $K_{s / c}$, but restricted to small $|\bar{\lambda}|,|\lambda| \ll 1$. (Similar RG equations appear in the context of single wall carbon nanotubes, where also two spin-degenerate channels are present 29.)

\section{B. Fixed points for the renormalization group equations}

The following types of fixed points are found for the RG equations (45)-(48):

- Fixed points where $K_{s} \neq 0$. As evident from (47), for these fixed points $\lambda=\bar{\lambda}=0$, and thus we refer to them as the "weak coupling fixed points". They describe wires with vanishing backscattering interaction at low temperatures, and therefore vanishing drag. As in the spin polarized case, for large values of $K_{c}, K_{s}$ these fixed points are stable, while for small values they are unstable.

- Fixed points for which $K_{s}=0$ and $K_{c} \neq 0$. As evident from (48), for these points $\bar{\lambda}=0$, and then, from (46), a stable fixed point exists only for $|\lambda|=\infty$. The former, $\bar{\lambda}=0$, indicates vanishing drag, the latter, $|\lambda|=\infty$, means that the spinmodes are massive (spin gap). These fixed points have no analog in the spin-less case. We refer to them as the "spin-gap fixed points".

- Fixed points where $K_{s}=K_{c}=0$. There are two stable fixed points in this plane. The first is $\lambda=-\infty$ and $\bar{\lambda}=+\infty$, and it obviously describes two strongly coupled wires, with diverging zero temperature drag. We refer to it as the "strong coupling fixed point". The second is a spin-gap fixed point as described above. There are also two unstable fixed points on that plane. The first is $\lambda=\bar{\lambda}=0$. It is repulsive in all directions of the $(\lambda, \bar{\lambda})$ plane. The second is $\lambda=\bar{\lambda}=1$. It is attractive in the direction $(1,1)$. The border between the basin of attraction of the two stable fixed points is the diagonal $\lambda=\bar{\lambda}$ (see Fig. 2). This border has a simple physical interpretation, separating between the region where bare intra-wire backscattering is stronger than the bare inter-wire one and the region where the opposite is true.

\section{The small $U$ limit}

In case of small $U_{c / s}$ Eqs. (45) to (48) reduce to the following set of equations, derived first by Gorkov and Dzyaloshinskii for the problem of coupled chains [30,14,

$$
\begin{aligned}
\frac{d \bar{\lambda}}{d x} & =-\left(U_{s}+U_{c}+2 \lambda\right) \bar{\lambda}, \\
\frac{d \lambda}{d x} & =-2 U_{s} \lambda-2 \bar{\lambda}^{2}, \\
\frac{d U_{s}}{d x} & =-2\left(\bar{\lambda}^{2}+\lambda^{2}\right), \\
\frac{d U_{c}}{d x} & =-4 \bar{\lambda}^{2},
\end{aligned}
$$

$\left(U_{c / s} \equiv U_{c-/ s \pm}\right)$. Furthermore, in that limit $\lambda_{0}=U_{s}^{0}$, and $d\left(\lambda-U_{s}\right) / d x \equiv 0$. Therefore $\lambda(x)=U_{s}(x)$. With this, Eqs. (49) reduce to three independent equations:

$$
\begin{aligned}
\frac{d \bar{\lambda}}{d x} & =-\left(U_{c}+3 \lambda\right) \bar{\lambda}, \\
\frac{d \lambda}{d x} & =-2\left(\lambda^{2}+\bar{\lambda}^{2}\right), \\
\frac{d U_{c}}{d x} & =-4 \bar{\lambda}^{2} .
\end{aligned}
$$

These RG flow equations were analyzed in detail by Lee et al. [14], who found that for initial values satisfying

$$
U_{c} \lambda<\bar{\lambda}^{2}
$$

the system scales to a strong coupling fixed point $\lambda \rightarrow$ $-\infty, \bar{\lambda} \rightarrow \infty$. Rewritten in terms of $g_{i \ldots}, \bar{g}_{i \ldots}$ this condition states

$$
\bar{g}_{2}-g_{2}<\frac{1}{2}\left(\frac{\bar{g}_{1}^{2}}{g_{1}}-g_{1}\right)+O\left(g_{i \ldots}^{2} / v_{F}^{2}\right)
$$

For $g_{1}>0$ the r.h.s. is always larger than $\bar{g}_{1}-g_{1}$, such that a sufficient condition for this inequality to be fulfilled is $\bar{g}_{2}-g_{2}<\bar{g}_{1}-g_{1}$. This, however, is exactly the inequality (13) we had in the spin-less case. We find then again that for weak monotonously decaying repulsive electronelectron interaction (small positive $\lambda$ ), a double wire with spin unpolarized electrons scales towards strong coupling, manifested by diverging trans-resistance at zero temperature. The condition $\lambda_{0}=U_{s}^{0}$, valid in the small $U$ regime, does not allow a flow to the spin-gap fixed point. 


\section{Beyond the small $U$ limit - linear stability of the weak coupling fixed points}

As stated above, a small $U$ analysis (i.e., weak electron-electron interaction) of the RG equations (45)(48) leads to the conclusion that $\bar{\lambda}$ and therefore also the drag resistivity diverges at zero temperature, accompanied by a large negative coupling $\lambda$. It is unlikely that stronger electron-electron interaction would push the system towards the weak coupling fixed points, where both backscattering interactions become negligible at zero temperature. It is, however, conceivable that for some range of parameters strong interaction would lead the system towards the spin-gap fixed point, where $\lambda$ goes to large positive coupling, but $\bar{\lambda}$ (and $\rho_{D}$ ) vanishes. For example, if in the absence of inter-wire coupling each of the wires is in a spin-gapped state with $\bar{\lambda} \gtrsim 1$ (as happens for attractive intra-wire electron-electron interaction) one may expect weak inter-wire coupling to leave the two wires in that state. Consequently, the wires would decouple $(\bar{\lambda} \rightarrow 0)$ and the drag would vanish.

In principle one may analyze this behavior by use of the RG equations (45)-(48) beyond the small $U$ limit. The validity of such an analysis to the problem at hand is however unclear, since at the level of initial values $\lambda_{0}>U_{s}^{0}$, and the RG equations are derived under the assumption of small $\lambda$. Instead of doing this, we treat $K_{c / s}, \lambda$ and $\bar{\lambda}$ as independent parameters and confine ourselves to the case of small couplings $\bar{\lambda}, \lambda \ll 1$ (but arbitrary $K_{c / s}$ ), where the RG equations are valid.

Let us consider the linear stability of the weak coupling fixed points (where $\lambda=\bar{\lambda}=0$ ) to turning on a small $\lambda, \bar{\lambda}$. We find that the $\lambda=\bar{\lambda}=0$ plane is split into four regions (see Fig. 3). In the first region, area $I$, defined by $K_{s}+K_{c}>2$ and $K_{s}>1$, the weak coupling fixed point is stable. In the region where $K_{s}>1$ but $K_{s}+K_{c}<2$ (which we call area $I I$, see Fig. 3), the weak coupling fixed points are linearly stable with respect to infinitesimal values of $\lambda$, but unstable with respect to such values of $\bar{\lambda}$. We now argue that initial values in that area flow to the strong coupling fixed point $(\lambda \rightarrow-\infty, \bar{\lambda} \rightarrow+\infty)$. Let us consider $\left|\bar{\lambda}_{0}\right| \ll \lambda_{0} \ll K_{s}-1$ in this region. By Eq. (46), the coupling constant $\lambda$ will scale to small values, such that the instability of the $\bar{\lambda}$-mode (Eq. 45) becomes relevant. Then $\bar{\lambda}$ keeps increasing until finally the $-2 \bar{\lambda}^{2}$-term in Eq. (46) overcomes the $\lambda$-stability and forces $\lambda$ to scale to negative values.

Points lying below the $K_{s}=1$ line in area $I I I$ or $I I I^{\prime}$ are linearly unstable in $\lambda$. Consequently, $\lambda$ increases and thereby decreases the coefficient in the equation for $\bar{\lambda}$. In the limit of $\bar{\lambda}_{0} \ll \lambda_{0}$ this leads to a stabilization of the $\bar{\lambda}$-mode, even where it was initially unstable (area $I I I^{\prime}$ ). Hence, points in areas $I I I$ and $I I I^{\prime}$ scale to the spin-gap fixed point $(\lambda \rightarrow+\infty, \bar{\lambda}=0)$.

To summarize, in the limit of $|\bar{\lambda}| \ll \lambda \ll\left|K_{s}-1\right|$ the basins of attraction of the weak and strong coupling fixed points correspond to areas I and II in Fig 3, respectively. Further, points of area $I I I / I I I^{\prime}$, which characterize systems with tendency to (single-wire) spin gap phase, indeed scale towards the (double-wire) spin-gap fixed point with vanishing $\bar{\lambda}$ (zero drag).

The linear stability analysis we carried out is expected to give the right structure of Fig. (33), but not the precise borders between the areas. Near the borders terms linear in $\lambda$ and $\bar{\lambda}$ may have very small prefactors, which make the role of the quadratic terms significant. An example to that is the region $K_{s} \approx 1+\lambda$, studied by Lee et al., where in the right hand side of (46) both terms become comparable.

When $\lambda_{0}$ is comparable to $\left|K_{s}-1\right|$ (but still $\bar{\lambda}_{0} \ll 1$ ) the condition for $\lambda$ scaling towards weaker couplings is $K_{s}-1>\lambda_{0}$ (see the discussion in Sect. IIII). Then, the border between areas $I I I / I I I^{\prime}$ and $I I / I$ is raised to $K_{s}=1+\lambda_{0}$. The effect of larger $\lambda_{0}$ is therefore a reduction of area $I I$, i.e. also in this sense a larger $\lambda_{0}$ is in favor of wire decoupling.

\section{E. High and low temperature limits of the drag}

A perturbative calculation of the drag-voltage in the weak coupling regime, applicable for widely separated wires in the high temperature regime, can be developed along the same lines as before. We have

$$
e V_{w}=\frac{a \kappa}{\pi}\left\langle\partial_{x}^{2} \phi_{c+}+w \partial_{x}^{2} \phi_{c-}\right\rangle_{I}
$$

The transformation to current carrying states is again given by (16), such that

$$
\begin{aligned}
e V_{w} & =w \frac{a \kappa}{\pi}\left\langle\partial_{x}^{2} \phi_{c-}\right\rangle_{I} \\
& =w \frac{4 a \kappa}{\pi \alpha^{2}} \bar{\lambda}\left\langle\sin \left(2 \phi_{c-}+\sqrt{2} \Omega t\right)\left(\cos 2 \phi_{s-}+\cos 2 \phi_{s+}\right)\right\rangle .
\end{aligned}
$$

Hereby, we used the Eq. of motion for $\phi_{c-}$ and the stationarity condition $\left\langle\partial_{t}^{2} \phi_{c-}\right\rangle=0$. A perturbative expansion in the couplings $\bar{\lambda}, \lambda$ and $U_{s} \approx \lambda$ then leads to

$$
\rho_{w}=w \rho_{0} \bar{\lambda}^{2}\left(\frac{T}{E_{0}}\right)^{2 K_{c}-1}
$$

with $\rho \sim h / e^{2} \alpha$. Leading higher order terms are of order $\bar{\lambda}^{2} \times O\left(\lambda, U_{s}\right)$.

For wire distances $\bar{d} \gg k_{F}^{-1}$ the smallness of $\bar{\lambda} \propto$ $e^{-2 k_{F} \bar{d}}$ is guaranteed. Further, in this case the spinsector couplings $\lambda, U_{s}$ flow to the single wire Luttinger Liquid fixed point $\lambda^{*}=U_{s}^{*}=0$, as long as the temperature is still large compared to $T^{*}$. Hence, also effective couplings $\lambda \approx U_{s}$ are small, wherefore the perturbative result (52) is applicable. 
The cross-over temperature $T^{*}$, separating between the high and low temperature regimes, is exponentially suppressed for $\bar{d} \gg k_{F}^{-1}$ with

$$
T^{*} \sim E_{0} \exp \left(-\frac{2 k_{F} \bar{d}}{1-K_{c}}\right)
$$

as shown in appendix A.

When the system flows towards the strong coupling fixed points, at sufficiently low temperatures the phases $\phi_{s \pm}, \phi_{c-}$ freeze to their minimum positions, and antisymmetric current flows by means of solitons. Again, in this case we expect drag resistivity to be proportional to $e^{T * / T}$.

\section{ESTIMATE OF PARAMETERS}

The significance of Coulomb drag in a particular experimental set-up of two 1D coupled wires is determined by $T^{*}$ and the corresponding minimal length $L^{*}=\alpha E_{0} / T^{*}$. We now estimate these quantities for a double wire system with parameters taken from the experiment by Yacoby et al. 21, where, using cleaved edge over-growth in GaAs-AlGaAs structures, quantum wires of width down to $14 \mathrm{~nm}$ and length $L$ of order $\mu \mathrm{m}$ were fabricated, with adjustable electron density.

The bare values of the interaction constants $g_{i \ldots}, \bar{g}_{i \ldots}$ appearing in the fermionic Hamiltonians can be estimated from the geometry of the experiment. If we assume the wires to be parallel in a distance $\bar{d}$ and characterize the transversal extension of the electron wavefunction by a length $d$, intra- and inter-wire potential are given by

$$
V(x)=\frac{e^{2}}{\epsilon}\left(x^{2}+d^{2}\right)^{-1 / 2}, \quad \bar{V}(x)=\frac{e^{2}}{\epsilon}\left(x^{2}+d^{2}+\bar{d}^{2}\right)^{-1 / 2} .
$$

The expression for $V(x)$ is approximate on small lengthscales $\lesssim d$. However, since generally $k_{F}^{-1} \gtrsim d$, this may not lead to large errors in the determination of the $2 k_{F}$ scattering parameters, $g_{1}, \bar{g}_{1}$. The couplings $g_{i}, \bar{g}_{i}$, are the Fourier components of $V(x), \bar{V}(x)$ at $q_{1}=2 k_{F}$ and $q_{2}, q_{4} \rightarrow 0$,

$$
g_{i}=\frac{2 e^{2}}{\epsilon} \mathrm{K}_{0}\left(q_{i} d\right), \quad \bar{g}_{i}=\frac{2 e^{2}}{\epsilon} \mathrm{K}_{0}\left(q_{i} \sqrt{d^{2}+\bar{d}^{2}}\right) .
$$

Taking the limit $q_{1} \rightarrow 0$ does not raise any problem, since the $q \rightarrow 0$ couplings $g_{2 / 4}, \bar{g}_{2 / 4}$ appear always as differences. Screening by metallic gates used in the experiment is negligible due to the relative large distance. As pointed out by Starykh et al. ( [31], see also Mahan [32]), the parameter $g_{4}$ has to be modified for the Pauli principle to be satisfied. This modification is however small when the $2 k_{F}$ part of the interaction is much smaller than the $q \approx 0$ part.
Having determined $g_{i \ldots}, \bar{g}_{i \ldots}$, we can calculate the bare parameters $\lambda_{0}, K_{c-}^{0}$, and $\bar{\lambda}_{0}, K_{s}^{0}$ for the bosonic Hamiltonians by means of the corresponding expressions of Sect. III and IV. These values are then to be used as initial values $(x=0)$ for the $\mathrm{RG}$ equations.

The system enters the regime of strong coupling when $\lambda(x) \sim 1$ (spin-polarized) or $\bar{\lambda}(x) \sim 1$ (spin-unpolarized). Thus, $T^{*}$ and $L^{*}$ are estimated by $\lambda\left(x^{*}\right)=1$ or $\bar{\lambda}\left(x^{*}\right)=$ 1 , respectively. The integration of the $\mathrm{RG}$-equations can be done analytically in certain parameter regimes, which leads to the expressions (22), (23), and (53) (see appendix A). Here we also give numerical results.

One should be aware that this method leads to order of magnitude estimates rather than precise values for $T^{*}$. Nevertheless, the reliability of this procedure can be demonstrated in the special case of spin-polarized fermions with $K_{c-}=1 / 2$. Then, the bosonic Hamiltonian $H_{c-}$ can be transformed to a Hamiltonian of noninteracting, fictitious fermions ("refermionization") [16], which exhibit an energy gap of the order of $T^{*}$ obtained by the aforementioned method (see appendix Q).

Let us first consider the spin-polarized case. We assume an inter-wire distance of $\bar{d}=3 d$. Table 田 contains $\lg _{10} E_{0} / T^{*} \equiv \lg _{10} L^{*} / r$ for wire widths $d=a_{B}, 2 a_{B}$ and $4 a_{B}$, where $a_{B}$ is the effective Bohr radius. With $a_{B} \approx 10 \mathrm{~nm}$ in GaAs these wire widths are close to the experimental values in [21]. The TL-parameter of the relative charge sector is typically $K_{c-} \approx 0.8$ and $\lambda_{0} \lesssim 0.1$. The main characteristic is the strong decrease of $x^{*}$ with $r$ as long $r \lesssim \bar{d}$, in accordance with the exponential suppression of $T^{*}$ (see Eq. (22)). For larger values of $r$ the dependence is rather weak, since here, according to (23), $x^{*} \approx \pi^{3} c\left(k_{F}\right) / r_{s} k_{F} \bar{d}$ with $c\left(k_{F}\right) / r_{s} k_{F} \approx$ const..

The strong coupling regime is not easily accessible. Even at very low densities of $r^{-1}=(200 \mathrm{~nm})^{-1}$ the crossover temperature is still very low: $T * \sim 0.01 E_{0} \sim$ $1 \mathrm{mK}$ (the corresponding length is $L^{*} \sim 10 \mu \mathrm{m}$ ). However, even when the system is still in the weak coupling regime, the drag resistance can be significant. According to the Eq. (20) we estimate the drag resistance of a $10 \mu$ long double wire at $T=100 \mathrm{mK}$ with $d=10 \mathrm{~nm}, \bar{d}=3 d$, and $r / a_{B}=5,10$ and 20 to be of order $0.01,0.1$ and $1 \times h / e^{2}$, respectively.

Quantitatively, our findings deviate strongly from the estimate for $L^{*}$ given by Nazarov and Averin [13]. For parameters $d=\bar{d}=10 \mathrm{~nm}, D=100 \mathrm{~nm}$ Ref. [13] obtain $L^{*} \approx 0.3 \mu \mathrm{m}$, whereas according to our calculations for these parameters $L^{*} \approx 1000 \mu \mathrm{m}$. The origin of this discrepancy of more than three orders of magnitude is explained in the next section.

The results obtained for a double wire of spinunpolarized electrons is shown in Tab. II (still $\bar{d}=3 d$, $D=200 \mathrm{~nm}$ ). For relatively small values $r \lesssim \bar{d}$ the system scales to strong inter wire couplings $\bar{\lambda}$. In this regime, $x^{*}$ decreases with increasing $r$, as in the previous 
case. A qualitatively different behavior sets in at larger $r$ : here, renormalization drives the system towards the spin gap fixed point, indicated by a "*" in Tab. II. The transition from the former regime to the latter happens at $r_{c} / a_{B}=3.1,4.8$, and 7.6 for $d / a_{B}=1.0,2.0$, and 4.0, respectively. At these densities, the TL parameter assume values $K_{c-} \approx 0.5$ and $K_{s} \approx 1.3$. Since further $\lambda \approx 0.3$, it is unclear, however, to what extent the $\mathrm{RG}$ equations we use, which are derived for small $\lambda_{0}, \bar{\lambda}_{0}$ are applicable for this case.

\section{SUMMARY}

In this paper we used the Tomanaga-Luttinger model to analyze Coulomb drag between two ballistic quantum wires. We find the drag to be a strong effect, both in its magnitude and in its temperature dependence.

We find that at zero temperature, for all weak monotonously decaying repulsive electron-electron interaction the trans-resistance diverges, indicating the formation of inter-locked charge density wave ground state in the two wires. At low temperature, $T \ll T^{*}$, we predict the trans-resistance to depend exponentially on $T^{*} / T$.

At high temperature $T \gg T^{*}$ we predict the transresistance to show a power law dependence on temperature, with the power being determined by the Luttinger liquid parameters. For spin polarized electrons, we find the power to be $4 K_{c-}-3$, with $K_{c-}$ being the TLparameter corresponding to anti-symmetric charge displacement. In this, our findings are in contrast to that of Ref. 13] where the exponent was identified with the parameter corresponding to symmetric charge displacement. Quantitatively, the two parameters are different, and this difference leads to the big difference in the estimates for $T^{*}$. For spin unpolarized electrons, we find the power to be $2 K_{c-}-1$.

The crossover temperature $T^{*}$ depends exponentially on parameters. For wires at large separation $k_{F} \bar{d} \gg 1$ it is $T^{*} \sim E_{0} \exp -\frac{b k_{F} \bar{d}}{1-K_{c-}^{0}}$, where $b=1$ for spinunpolarized, and $b=2$ for spin-polarized wires.

We thank I. Aleiner, A. Finkel'stein, D. Maslov, A. Punnoose and Y. Oreg for instructive discussions, and particularly A. Moustakis, L. Balents and S. Simon for a discussion of the RG equations. We thank the MinervaFoundation (Munich), the Bi-national Israel-USA foundation, the Israel academy of Science and the Victor Ehrlich chair for financial support.

\section{APPENDIX A:}

We begin with the calculation of $T^{*}$ for spin-less electrons and large wire separation $\bar{d}$, such that $k_{F} \bar{d} \gg 1$. In this case

$$
\lambda_{0}=\frac{2 e^{2}}{\pi \epsilon u_{c-}} \mathrm{K}_{0}\left(2 k_{F} \bar{d}\right) \sim r_{s} e^{-2 k_{F} \bar{d}}
$$

is exponentially small, as a consequence of which $K_{c-}(x) \approx$ const. $\equiv K_{0}$ (Eq. 11). With this we obtain from $\mathrm{d} \ln \lambda / \mathrm{d} x=2-2 K_{0}$

$$
\lambda(x)=\lambda_{0} e^{\left(2-2 K_{0}\right) x},
$$

where $x=\ln E_{0} / T$. The condition $\lambda\left(x^{*}\right) \approx 1$ then gives

$$
T^{*} \approx E_{0} \lambda_{0}^{1 /\left(2-2 K_{0}\right)} \sim E_{0} \exp \left(-\frac{k_{F} \bar{d}}{1-K_{0}}\right) .
$$

For small wire separations $(d \approx \bar{d})$ inter and intra wire couplings $\bar{g}_{\ldots}$ and $g_{\ldots}$ become similar, such that $\left(U_{0} \equiv U_{c-}^{0}\right)$

$$
U_{0}=\frac{1}{2 \pi v_{c-}}\left(g_{1}-g_{2}+\bar{g}_{2}\right) \approx \frac{g_{1}}{2 \pi v_{c-}} \approx \frac{\bar{g}_{1}}{2 \pi u_{c-}}=\lambda_{0} .
$$

This means that the bare couplings $\lambda_{0}, K_{0} \approx 1+U_{0}$ lie close to the separatrix, but still outside the attractive region of the weak coupling fixed points (see Fig. 11). Under renormalization $(\lambda(x), K(x))$ flows along the separatrix towards weaker couplings until the turning point at $K=1$ is reached. Thenceforward $(\lambda, K)$ flows towards the strong coupling regime.

For an estimate of $T^{*}$ we use the approximate $\mathrm{RG}$ equations valid for $|U| \ll 1$,

$$
\frac{\mathrm{d} \lambda}{\mathrm{d} x}=-2 \lambda U, \quad \frac{\mathrm{d} U}{\mathrm{~d} x}=-2 \lambda^{2} .
$$

These can be easily integrated by use of the constant of motion $\lambda^{2}-U^{2} \equiv a^{2}$, which yields

$$
x_{2}-x_{1}=\frac{1}{2 a}\left(\arctan U_{1} / a-\arctan U_{2} / a\right) .
$$

In particular, we obtain for the "time" $x_{a}$, at which the turning point is reached $\left(x_{1}=x_{0}=0, U_{1}=U_{0}, x_{a}=\right.$ $\left.x_{2}, U_{2}=0\right)$

$$
x_{a}=\frac{1}{2 a} \arctan U_{0} / a .
$$

In the case under consideration with $\lambda_{0} \approx U_{0}$, the constant $a$ is a small quantity:

$$
a^{2} \approx 2 \lambda_{0}\left(\lambda_{0}-U_{0}\right) \ll \lambda_{0}^{2}, U_{0}^{2},
$$

which will be determined more explicitly later. Because of the relative smallness of $a$ we have

$$
x_{a} \approx \frac{\pi}{4 a} .
$$

For the rise from the turning point to stronger couplings $\lambda \gg a$, an equally long "time" $x_{b}$ is needed, so that

$$
x^{*} \approx x_{a}+x_{b}=\pi / 2 a
$$


and therefore

$$
T^{*} \approx E_{0} e^{-\pi / 2 a} .
$$

It remains to calculate $a$ : Taking the expressions

$$
g_{i}=\frac{2 e^{2}}{\epsilon} \mathrm{K}_{0}\left(q_{i} d\right), \quad \bar{g}_{i}=\frac{2 e^{2}}{\epsilon} \mathrm{K}_{0}\left(q_{i} \bar{d}\right),
$$

and expanding up to second order in $k_{F} \bar{d}$ we obtain

$$
\begin{aligned}
\lambda_{0}-U_{0} & =\frac{1}{2 \pi v_{c-}}\left(\bar{g}_{1}-g_{1}+g_{2}-\bar{g}_{2}\right) \\
& =\frac{r_{s}}{\pi^{2}} f\left(d / \bar{d}, \ln k_{F} \bar{d}\right)\left(k_{F} \bar{d}\right)^{2}+O^{4}\left(k_{F} \bar{d}\right),
\end{aligned}
$$

whereby

$$
f\left(d / \bar{d}, \ln k_{F} \bar{d}\right)=\left(\frac{d}{\bar{d}} \ln d / \bar{d}+\gamma-1+\ln k_{F} \bar{d}\right)\left(\frac{d^{2}}{\bar{d}^{2}}-1\right) .
$$

( $\gamma \approx 0.577$ is Euler's constant) This yields

$$
a=\left(2 \lambda_{0}\left(\lambda_{0}-U_{0}\right)\right)^{1 / 2}=\frac{r_{s}}{\pi^{2}} \mathrm{~K}_{0}^{1 / 2}\left(2 k_{F} \bar{d}\right) f^{1 / 2} k_{F} \bar{d} .
$$

As long $k_{F} \bar{d} \lesssim 1$, the Bessel-function $\mathrm{K}_{0}\left(2 k_{F} \bar{d}\right)$ and $f$ are of order one and only logarithmically dependent on $k_{F} \bar{d}$. Hence in this case

$$
a=c^{-1} \frac{r_{s}}{2 \pi^{2}} k_{F} \bar{d}
$$

with $c$ a numerical coefficient of order unity. Inserting this into A3 yields

$$
T^{*} \sim E_{0} \exp \left(-\frac{c \pi^{3}}{r_{s} k_{F} \bar{d}}\right) .
$$

Let us now consider a spin-full double wire with large separation $\bar{d} \gg k_{F}^{-1}$. Then again the inter wire backscattering coupling is exponentially small, such that also here

$$
K_{c} \approx \text { const. }=K_{c}^{0} .
$$

Due to the smallness of $\bar{\lambda}$, the RG equations for the spinsector couplings $\lambda, K_{s}$ decouple from the relative charge sector and become identical to the corresponding $\mathrm{RG}$ equations of a single spin- $\frac{1}{2}$ wire:

$$
\begin{aligned}
\lambda^{\prime} & =-2 U_{s} \lambda, \\
U_{s}^{\prime} & =-2 \lambda^{2}
\end{aligned}
$$

(for $\lambda,\left|U_{s}\right| \ll 1$ ). Accordingly, the couplings $\lambda, U_{s}$ with bare values $\lambda_{0}=U_{s}^{0}=g_{1} / 2 \pi v_{F}$ scale down to weaker coupling:

$$
\lambda(x)=U_{s}(x)=\frac{\lambda_{0}}{1+2 \lambda_{0} x} .
$$

Because of this behavior we can neglect in the differential equation for $\bar{\lambda}$,

$$
\frac{\mathrm{d} \ln \bar{\lambda}}{\mathrm{d} x}=1-K_{c}^{0}-U_{s}-2 \lambda,
$$

the last two terms, if $U_{s}^{0}, \lambda_{0} \ll 1-K_{c}^{0} s$. Then, for this regime,

$$
\frac{\mathrm{d} \ln \bar{\lambda}}{\mathrm{d} x} \approx 1-K_{c}^{0}
$$

which gives

$$
\bar{\lambda}(x)=\bar{\lambda}_{0} e^{\left(1-K_{c}^{0}\right) x} .
$$

This leads in the same way as in the corresponding spinless case to a crossover temperature

$$
T^{*} \sim E_{0} \exp \left(-\frac{2 k_{F} \bar{d}}{1-K_{c}^{0}}\right) .
$$

This result deviates from the former estimation for the spin-less case only by a factor 2 in front of $k_{F} \bar{d}$. This extra factor reflects the fact that here the mean electron distance $\bar{r}$ is exactly half of the one in a spin-less wire, when the Fermi momentum is the same.

\section{APPENDIX B: RELATION TO THE MEMORY-FUNCTION FORMALISM}

In this section we elaborate on the relation of the perturbative calculation of subsection IIID and a formula for the Coulomb drag resistivity based on the memoryfunction formalism by Zheng and MacDonald (Eq. (12) in 25]).

From Eqs. (18) and (19) follows that

$$
\rho=M \frac{\partial}{\partial \Omega}\left\langle\frac{\delta H_{\text {int }}(\Omega)}{\delta \phi\left(x_{0}\right)}\right\rangle,
$$

where $M$ is a constant factor determined by the system parameters, and $H_{\text {int }}$ is given by Eq. (17). A first order expansion in $H_{\text {int }}=H_{\text {int }}(\Omega)$ leads to

$$
\left\langle\frac{\delta H_{\text {int }}}{\delta \phi\left(x_{0}\right)}\right\rangle \approx i \int_{0}^{\infty} d t\left\langle\left[H_{\text {int }}(t), \frac{\delta H_{\text {int }}(t=0)}{\delta \phi\left(x_{0}\right)}\right]\right\rangle_{0} .
$$

where the subscript 0 indicates a thermal average taken with respect to $H_{0}$. For $I \propto \Omega \rightarrow 0$ we can expand $H_{\text {int }}$ in $\Omega$,

$$
H_{i n t}(\Omega) \approx H_{i n t}(\Omega=0)+\frac{\Omega t}{\sqrt{2}} \int \frac{\delta H_{i n t}}{\delta \phi(x)} d x,
$$

and obtain

$$
\rho=i \frac{M}{\sqrt{2}} \int_{0}^{\infty} d t t \int d x\left\langle\left[\frac{\delta H_{i n t}(t)}{\delta \phi(x)}, \frac{\delta H_{i n t}(0)}{\delta \phi\left(x_{0}\right)}\right]\right\rangle_{0} .
$$

Then, making use of the Kubo-identity 


$$
\begin{aligned}
{\left[e^{-\beta H_{0}}, A\right] } & =i e^{-\beta H_{0}} \int_{0}^{\beta} d \lambda \frac{d A}{d t}(-i \lambda) \\
& =i e^{-\beta H_{0}} \int_{0}^{\beta} d \lambda e^{\lambda H_{0}} \frac{d A}{d t} e^{-\lambda H_{0}}
\end{aligned}
$$

and partial integration leads to

$$
\rho=\frac{M}{\sqrt{2}} \int_{0}^{\infty} d t \int_{0}^{\beta} d \lambda \int d x\left\langle\frac{\delta H_{\text {int }}(-i \lambda)}{\delta \phi\left(x_{0}\right)} \frac{\delta H_{i n t}(t)}{\delta \phi(x)}\right\rangle_{0} .
$$

Since the functional derivatives of $H_{\text {int }}$ are the intrawire force-densities (up to a constant factor), this expression essentially equals the corresponding formula (12) of Zheng and MacDonald.

\section{APPENDIX C: REFERMIONIZATION}

Luther and Emery [16] observed that in the special case $K_{c-}=1 / 2$ the bosonic Hamiltonian $H_{c-}$ can be mapped onto an exactly solvable Hamiltonian of fictitious, noninteracting fermions ("refermionization"). After the canonical transformation $\Pi_{c-}, \phi_{c-} \rightarrow \Pi^{\prime}=2^{1 / 2} \Pi_{c-}, \phi^{\prime}=$ $2^{-1 / 2} \phi_{c-}$ the Hamiltonian states

$$
H_{c-}=\frac{u_{c-}}{2 \pi} \int d x \pi^{2} \Pi^{\prime 2}+\left(\partial_{x} \phi^{\prime}\right)^{2}+\frac{2 \bar{g}_{1}}{2 \pi \alpha} \int d x \cos \left(2 \phi^{\prime}\right),
$$

which expressed by fermions

$$
\begin{aligned}
a_{r}(k) & =2^{-1 / 2} \int d x \psi_{r}(x) e^{-i k x} \\
\psi_{r} & =(2 \pi \alpha)^{-1 / 2} \exp \left(i r\left(k_{F} x+\phi^{\prime}\right)+i \pi \int_{x_{0}}^{x} d x^{\prime} \Pi^{\prime}\left(x^{\prime}\right)\right),
\end{aligned}
$$

becomes

$$
\begin{aligned}
H_{c-}^{f}= & u_{c-} \sum_{r k} r k a_{r}^{\dagger}(k) a_{r}(k) \\
& +\frac{\bar{g}_{1}}{2 \pi \alpha} \sum_{k} a_{+}^{\dagger}(k) a_{-}\left(k-2 k_{F}\right)+h . c . .
\end{aligned}
$$

This Hamiltonian is diagonziable by a simple Bogoliubov transformation. The spectrum

$$
E_{ \pm, k}=u_{c-} k_{F} \pm \sqrt{\left(\frac{\bar{g}_{1}}{2 \pi \alpha}\right)^{2}+u_{c-}\left(k-k_{F}\right)^{2}}
$$

exhibits an energy gap of width $\Delta E=g / \pi \alpha$ at $\pm k_{F}$, which coincides up to a factor 2 with the corresponding temperature $T^{*}$ as estimated by integrating the RG flow: from Eq. (A2) with $K_{c-}=1 / 2$ we get

$$
T^{*}=E_{0} \lambda_{0}=E_{0} \frac{\bar{g}_{1}}{2 \pi u_{c-}}=\frac{\bar{g}_{1}}{2 \pi \alpha}=\frac{1}{2} \Delta E .
$$

[1] For a review, see A. G. Rojo, J. Phys. Condens Matter, 11, R31-R52 (1999), and references therein.

[2] S. Tomanaga, Prog. Theor. Phys. 5 ,544 (1950); J. M. Luttinger, J. Math. Phys. 4, 1154 (1963).

[3] F. D. M. Haldane, J. Phys. C 14, 2585 (1981); F. D. M. Haldane, Phys. Rev. Lett. 47, 1840 (1981).

[4] J. Voit, Rep. Prog. Phys 57, 977 (1994).

[5] V.J. Emery, Highly Conducting One-Dimensional Solids ed. J.T. Devreese, R.E. Evrard and V.E. von Doren, New York, Plenum (1979).

[6] J. Solyom, Adv. Phys. 28, 201 (1978).

[7] H. J. Schulz, G. Cuniberti, P. Pieri, Fermi liquids and Luttinger liquids Lecture notes of the Chia Laguna summer school, Italy (1997).

[8] Ben Yu-Kuang Hu and Karsten Flensberg, in Hot Carriers in Semiconductors, edited by K. Hess et al., Plenum Press, New York, 1996.

[9] O.E. Raichev, P. Vasilopoulos, Phys. Rev. Lett. 83, 3697 (1999).

[10] B. Tanatar, Phys. Rev. B 58, 1154 (1998).

[11] K. Flensberg, Phys. Rev. Lett. 81, 184 (1998).

[12] A. Komnik, R. Egger, Phys. Rev. Lett. 80, 2881 (1998).

[13] Yuli V. Nazarov, D. V. Averin, Phys. Rev. Lett. 81, 653 (1998).

[14] P.A. Lee, T.M. Rice, R.A. Klemm, Phys. Rev. B 15, 2984 (1977).

[15] A.M. Finkel'stein, A.I. Larkin, Phys. Rev. B 16, 10461 (1993); H.J. Schulz, Phys. Rev. B 53, R2959 (1996); L. Balents, M.P.A. Fisher, Phys. Rev. B 53, 12133 (1996); and references therein.

[16] A. Luther and V. J. Emery, Phys. Rev. Lett. 33, 589 (1974).

[17] S. T. Chui and P. A. Lee, Phys. Rev. Lett. 35, 315 (1975).

[18] M.J. Rice, A.R. Bishop, J.A. Krumhansl, S.E. Trullinger, Phys. Rev. Lett. 36, 432 (1976).

[19] K. Maki, Phys. Rev. Lett. 39, 46 (1977).

[20] S. Tarucha et al., Sol. State Comm. 94, 413 (1995).

[21] A. Yacoby et al., Phys. Rev. Lett. 77, 4612 (1996).

[22] J.V. Jose, L.P. Kadanoff, S. Kirkpatrick, D.R. Nelson, Phys. Rev. B 16, 1217 (1977).

[23] T. Giamarchi, H.J. Schulz, Phys. Rev. B 37, 325 (1988).

[24] In principle, also the conjugate field $\Pi_{+}$changes, nameley to $\Pi_{+}(x, t)+\frac{\Omega}{\pi v_{F}}$. However, $\Pi_{+}$appears to the second power in the kinetic energy term. Therefore the transformation of $\Pi_{+}$contributes to the drag only into second order in $I$ resp. $\Omega$. We will neglect this contribution from the very beginning by leaving $\Pi_{+}$unchanged.

[25] Lian Zheng and A. H. MacDonald, Phys. Rev. B 48, 8203 (1993).

[26] R. Rajaraman, Solitons and Instantons, North-Holland Publishing Company, Amsterdam (1982).

[27] A. Tsvelik, Quantum field Theory in Condensed Matter Physics, Cambridge University Press (1995).

[28] H. Kleinert, Pathintegrals in Quantum Mechanics, Statistics, and Polymer Physics, 2nd Edition, World Scientific Publishing, Singapore (1994). 
[29] R. Egger, A.O. Gogolin, Phys. Rev. Lett. 79, 5082 (1997), and Eur. Phys. J. B 3, 281 (1998); H. Yoshioka,A. A. Odintsov, Phys. Rev. Lett. 82, 374 (1999).

[30] L.P. Gor'kov, I.E. Dzyaloshinskii, Zh. Eksp. Teor. Fiz. 67, 397 (1974) [Sov. Phys.-JETP 40, 198 (1975)].

[31] O. A. Starykh, D. L. Maslov, W. Häusler and L. I. Glazman cond-mat/9911286, to appear in "Interactions and Transport Properties of Lower Dimensional Systems", Lecture Notes in Physics, Springer.

[32] G.D. Mahan, Many particle physics, 2nd edition, Plenum press, New-York, 1990, section 4.4 .

\begin{tabular}{c|ccc}
$d / a_{B}$ & 1.0 & 2.0 & 4.0 \\
\hline$r / a_{B}$ & & & \\
2.0 & 15 & 27 & - \\
3.0 & 8.2 & 14 & - \\
4.0 & 5.6 & 9.1 & 17 \\
5.0 & 4.3 & 6.7 & 11 \\
7.0 & 3.1 & 4.4 & 7.4 \\
9.0 & 2.6 & 3.3 & 5.3 \\
12. & 2.2 & 2.6 & 3.8 \\
16. & 2.0 & 2.1 & 2.8 \\
20. & 1.8 & 1.9 & 2.3
\end{tabular}

TABLE I. The table lists $\lg _{10} E_{0} / T^{*}$ (which is also $\left.\lg _{10} L^{*} / r\right)$ for different values of $d / a_{B}$ and $r / a_{B}$ for a spin-polarized double wire system $(\bar{d}=3 d, D=200 \mathrm{~nm}$, $\left.a_{B}=10 \mathrm{~nm}\right)$.

\begin{tabular}{l|ccc}
$d / a_{B}$ & 1.0 & 2.0 & 4.0 \\
\hline$r / a_{B}$ & & & \\
1.0 & 18 & - & - \\
1.5 & 11 & 18 & - \\
2.0 & 8.7 & 13 & 22 \\
2.5 & 7.6 & 9.9 & 17 \\
3.0 & 7.3 & 8.4 & 13 \\
3.5 & $*$ & 7.6 & 11 \\
4.0 & $*$ & 7.0 & 9.7 \\
4.5 & $*$ & 6.7 & 8.7 \\
5.0 & $*$ & $*$ & 8.0 \\
5.5 & $*$ & $*$ & 7.5 \\
6.0 & $*$ & $*$ & 7.1 \\
6.5 & $*$ & $*$ & 6.8 \\
7.0 & $*$ & $*$ & 6.6 \\
7.5 & $*$ & $*$ & 6.8 \\
8.0 & $*$ & $*$ & $*$
\end{tabular}

TABLE II. $\lg _{10} E_{0} / T^{*} \equiv \lg _{10} L^{*} / \alpha$ for a spin-unpolarized double wire system $\left(\bar{d}=3 d, D=200 \mathrm{~nm}, a_{B}=10 \mathrm{~nm}\right)$. The "*" indicates the zero-drag phase at $T=0$. Note that at large $r / a_{B}$ the initial values of $\lambda, \bar{\lambda}$ are not small, and thus the validity of the $\mathrm{RG}$ equations is questionable.

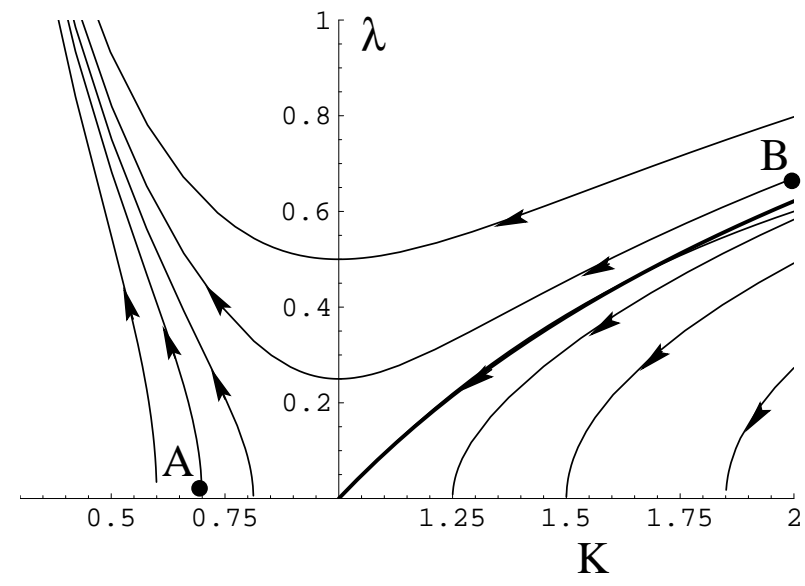

FIG. 1. The RG-flow of a double wire system of spin-less electrons. Point A corresponds to the bare couplings of a system with $\bar{d} \gg k_{F}^{-1}$, point $\mathrm{B}$ to wires with narrow spacing $\bar{d} \ll k_{F}^{-1}$.

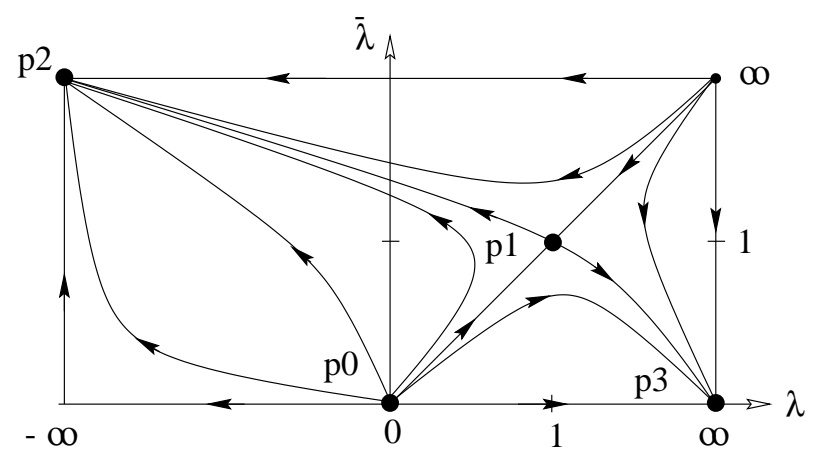

FIG. 2. RG flow of the spin-full double wire system in the $K_{c}=K_{s}=0$ plane (schematic). $p_{0}$ and $p_{1}$ are unstable fixed points, $p_{2}$ is the strong coupling fixed point, and $p_{3}$ a spin-gap fixed point. 


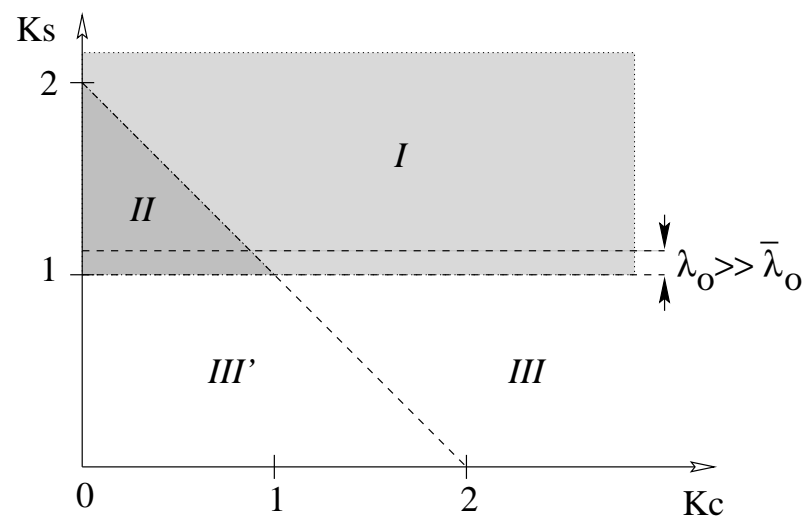

FIG. 3. Stability of weak coupling fixed points $\lambda=\bar{\lambda}=0$. Only fixed points in area $I$ can be stable. In the limit $\left|\bar{\lambda}_{0}\right| \ll \lambda_{0} \ll\left|K_{s}-1\right|$ points in area $I I$ flow to the strong coupling fixed point $p_{2}$ (where $\bar{\lambda} \rightarrow \infty$ ), while points in area $I I I$ or $I I I^{\prime}$ flow towards the spin-gap fixed point $p_{3}$ (where $\bar{\lambda} \rightarrow 0)$. 